\title{
Régulation des innovations dans la e-santé
}

Jean-Yves Fagon

> II paraît nécessaire de rappeler un certain nombre de principes de la part des pouvoirs publics. Tout d'abord, l'innovation se développe, avec diversité et, à grande vitesse. La sécurité des patients ne doit pas être remise en cause et au contraire être mise en place pour sécuriser un certain nombre d'innovations actuelles qui ont des particularités.

Un autre point est préoccupant. II porte sur l'égalité d'accès aux soins et à la santé. Dans ce cadre, les innovations peuvent être une fabrique d'inégalité. Cette question complexe doit être traitée. Sécurité et égalité d'accès sont des impératifs qui nécessitent de plus en plus des expertises, du temps et du travail.

Le troisième point porte sur les modalités de financement des innovations d'une manière générale et pour des projets qui suivent un régime particulier. Il existe de nombreux plans qu'il serait souhaitable de pouvoir développer dans les meilleures conditions possibles, mais qui peuvent être «ralentis » par une difficulté sur le plan financier.

On assiste aujourd'hui à l'arrivée massive d'innovations, notamment dans le champ des maladies rares. Certaines entraînent des évolutions importantes, pour lesquelles notre système n'est pas tout à fait adapté. Le nombre de demandes d'implantations ou d'accès à des innovations de tout type explose. À force de vouloir accélérer, nous pourrions finir par bloquer le développement de projets en nombre excessifs. Un certain nombre de porteurs de projets travaille sur des concepts similaires. Tout le monde est convaincu de proposer «l'innovation du siècle », mais dialoguer avec les acteurs d'autres régions permettrait d'éviter des pertes d'énergie et de temps. Il est impératif de parvenir à coordonner des acteurs travaillant sur des problématiques proches.

Par ailleurs, il n'y a quasiment plus d'innovation qui soit un seul produit de santé, dispositif, médicament ou organisation. Nous devons maintenant gérer une solution innovante combinant deux ou trois composantes, permettant à l'innovation d'être encore plus innovante et efficace. II faut donc adapter notre système car les innovations ainsi mises sur le marché sont d'une complexité importante. Intégrer cette complexité nécessite l'expertise de gens capables de l'analyser et de l'interpréter. Sur un certain nombre de rares sujets «pointus », il existe peut-être 4 ou 5 experts en France, qui ont des liens d'intérêt avec des sociétés qui ont participé au développement de ces innovations. Nous nous retrouvons donc actuellement dans une forme d'impasse, extrêmement limitante, et qu'il faut absolument dépasser. Nous devons pouvoir bénéficier de l'expertise de gens capables de donner la bonne interprétation et le bon développement d'un certain nombre de ces innovations.

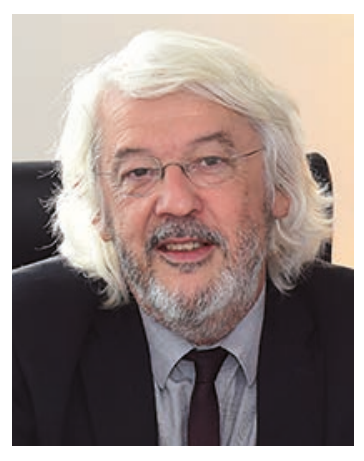

Délégué ministériel

à l'innovation en santé, Paris, France.

jean-yves.fagon@sg.social. gouv.fr

La lutte contre un développement imparfait des innovations en santé implique de coordonner les différents acteurs au sein des pouvoirs publics et avec les industriels concernés. II faut entrer dans la co-élaboration des innovations en continuant à faire avancer l'innovation ensemble. Nous gagnerons ainsi de l'efficacité et du temps et éviterons les dérives potentielles de certains porteurs de projet, le plus souvent en raison de la méconnaissance de la complexité de notre système. Aujourd'hui, la plupart des innovations, une fois à la disposition de la population, ne sont plus suivies. Nous manquons ainsi cruellement d'informations «en vie réelle». II faut assurer une période de suivi et d'évaluation après la mise sur le marché pour avoir le maximum de données permettant d'optimiser le développement de l'innovation dans des conditions les plus claires possibles. En implantant et facilitant la mise en place de cette stratégie concernant les innovations en santé, nous avons peut-être une chance d'en favoriser le développement de notre pays.

De nombreux projets portent sur l'amélioration de la sortie de l'hôpital des patients. II est vrai que la jonction Ville-Hôpital est médiocre. Les projets qui réussiront sont ceux qui se réuniront pour trouver la bonne solution et donner du sens en termes de parcours de soin. Les corporatismes sont malheureusement encore à l'œuvre. II faut une véritable volonté partagée par toutes les équipes et les pouvoirs publics pour faire fonctionner ce nouveau système au bénéfice des patients. $\diamond$

Regulation of innovations in the field of e-health

\section{LIENS D'INTÉRÊT}

L'auteur déclare n'avoir aucun lien d'intérêt concernant les données publiées dans cet article. 